\title{
A coming era of precision diagnostics based on nano-assisted mass spectrometry
}

\author{
Rongxin $\mathrm{Li}^{1 \dagger}$, Deepanjali Dattatray Gurav ${ }^{2 \dagger}$, Jingjing $\mathrm{Wan}^{1 *}$, and Kun Qian ${ }^{2 *}$ \\ ${ }^{I}$ School of Chemistry and Molecular Engineering, East China Normal University, Shanghai, 200062, P. R. \\ China \\ ${ }^{2}$ School of Biomedical Engineering, Med-X Research Institute, Shanghai Jiao Tong University, Shanghai, \\ 200030, P. R. China
}

Submitted: July 24, 2018;

†These authors contributed equally to this work.

Accepted: August 1, 2018

Published: August 21, 2018

\section{Keywords}

Diagnostics, mass spectrometry, nanotechnology, omics

\section{Abbreviations:}

- SNP single-nucleotide polymorphism

- qPCR quantitative real-time polymerase chain reaction

- a6A N6-allyladenosine

- RT reverse transcriptase

- CyTOF mass cytometry by time-of-flight

- Pre-DC dendritic cells precursors

\section{Abstract}

Precision diagnostics relies on omics analysis by mass spectrometry to overcome the limitation in accuracy by an individual biomarker, due to the complex nature of diseases. Recent development in nanotechnology markedly enhanced sample treatment and detection efficiency of this method. Herein, we foresee a coming era of precision diagnostics based on nano-assisted mass spectrometry. Some important progress in the field includes detection of (1) nucleic acids for genetic analysis; (2) proteins/peptides for proteomic analysis; and (3) small molecules for metabolic analysis. We anticipate that this review will be a reminder for both young and experienced researchers about the future of diagnostics and call for attention worldwide.

\section{Purpose and Rationale}

Diagnostics is the core of biomedical research and clinical practice, which guides the prevention and treatment of diseases for better healthcare globally. ${ }^{1-3}$ Notably, precision diagnostics relies on omic analysis to overcome the limitation in accuracy by an individual biomarker, ${ }^{4-7}$ due to the complex nature of physiological and pathological process. ${ }^{1,8,9}$ Among numerous analytical approaches for omics, mass spectrometry (MS) has become the major tool due to the desirable throughput, sensitivity, and identification capability. ${ }^{10-13}$ To date, the state-of-art MS methods and techniques have advanced omics in all levels including genomics, ${ }^{14,15}$ proteomics, ${ }^{16-18}$ and metabolomics. ${ }^{19-21}$

Nowadays, interdisciplinary research is fundamental to achieve real case applications of MS in clinics. Particularly, development of nanotechnology enhanced sample treatment and detection efficiency of MS for diagnostics. ${ }^{10,22-24}$ For sample treatment, nanoscale materials and devices afford unique size-dependent properties for selective extraction of either a specific molecule or a group of molecules. ${ }^{3,20,25,26}$ For detection efficiency, nanoscale materials and devices

\footnotetext{
*Corresponding Authors: E-mail: jjwan@chem.ecnu.edu.cn, k.qian@sjtu.edu.cn
} 
contribute to the amplified ionization performance with orders of magnitude. ${ }^{24,25,27}$ Therefore, nanotechnology can tackle the key obstacles for MS-based omics, which can be further combined with instrumentation and data mining for next generation of precision diagnostics.

Herein, we foresee a coming era of precision diagnostics based on nano-assisted MS. We show a few important developments in the field (Scheme 1 and Table 1), including MS detection of (1) nucleic acids for genetic analysis; (2) proteins/peptides for proteomic analysis; and (3) small molecules for metabolic analysis. We anticipated that this review would be a reminder for both young and experienced researcher about the future of diagnostics, calling for the attentions worldwide.

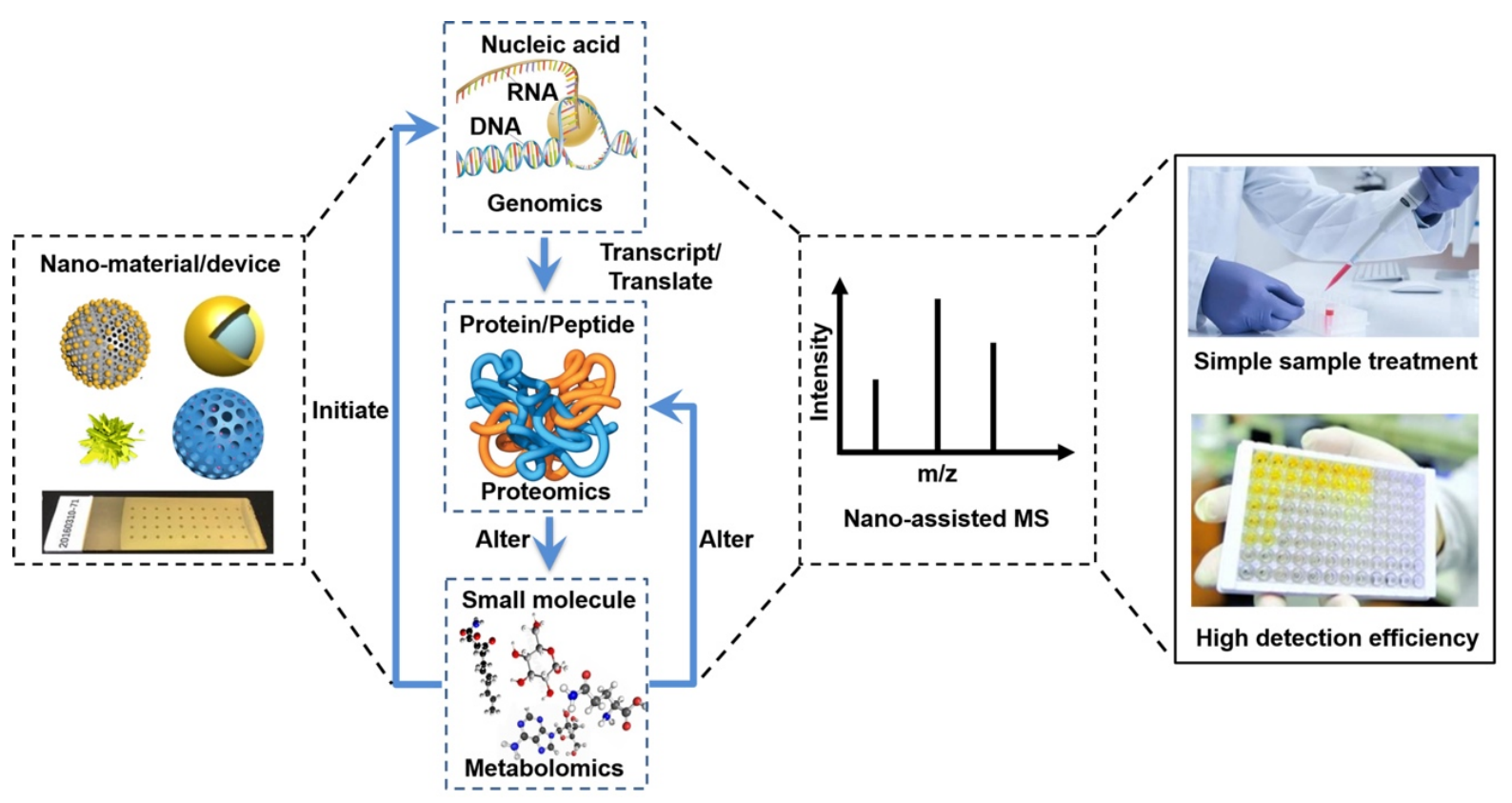

Scheme 1. Schematic illustration of omic analysis including genomics, proteomics, and metabolomics based on nanoassisted mass spectrometry.

Table 1 Overview of nano-assisted MS and typical applications that could be replaced

\begin{tabular}{|c|c|c|c|}
\hline $\begin{array}{l}\text { Categories for } \\
\text { nano-assisted } \\
\text { MS }\end{array}$ & $\begin{array}{l}\text { Performance of nano- } \\
\text { assisted MS }\end{array}$ & $\begin{array}{l}\text { Typical applications that could be } \\
\text { replaced }\end{array}$ & $\begin{array}{l}\text { Performance of typical } \\
\text { applications }\end{array}$ \\
\hline $\begin{array}{l}\text { Nano-MS } \\
\text { based genomics }\end{array}$ & $\begin{array}{l}\text { sensitivity: high } \\
\text { throughput: high } \\
\text { cost: moderate } \\
\text { quantitation: yes }\end{array}$ & $\begin{array}{l}\text { gene chip }^{[31]} \\
\text { quantitative real-time } \mathrm{PCR}^{[33]} \\
\text { sanger gene sequencing }\end{array}$ & $\begin{array}{l}\text { sensitivity: high } \\
\text { throughput: low } \\
\text { cost: very high } \\
\text { quantitation: yes }\end{array}$ \\
\hline $\begin{array}{l}\text { Nano-MS } \\
\text { based } \\
\text { proteomics }\end{array}$ & $\begin{array}{l}\text { sensitivity: high } \\
\text { throughput: high } \\
\text { cost: moderate } \\
\text { quantitation: yes }\end{array}$ & $\begin{array}{l}\text { chromatography }^{[38]} \\
\text { protein microarrays }^{[39]} \\
\text { gel electrophoresis }\end{array}$ & $\begin{array}{l}\text { sensitivity: high } \\
\text { throughput: moderate } \\
\text { cost: high } \\
\text { quantitation: yes }\end{array}$ \\
\hline $\begin{array}{l}\text { Nano-MS } \\
\text { based } \\
\text { metabolomics }\end{array}$ & $\begin{array}{l}\text { sensitivity: very high } \\
\text { throughput: very high } \\
\text { cost: low } \\
\text { quantitation: yes }\end{array}$ & $\begin{array}{l}\text { biochemical analyzer }^{[47]} \\
\mathrm{NMR}^{[48]} \\
\text { electrochemical sensing }^{[49]}\end{array}$ & $\begin{array}{l}\text { sensitivity: high } \\
\text { throughput: low } \\
\text { cost: moderate } \\
\text { quantitation: yes }\end{array}$ \\
\hline
\end{tabular}

MS = mass spectrometry; PCR = polymerase chain reaction . 


\section{Summary of relevant literature and discussion}

\section{Detection of nucleic acids for genetic} analysis

Genomics is the study of nucleic acids for genetic evaluation of an organism, analyzing the structure and function of genomes. ${ }^{28,29}$ Compared to the well-defined analysis of mutations at DNA level, the emerging fields of RNA editing, methylation, and splicing generate a library of transcript isoforms encoding genetic information from DNARNA-proteins. ${ }^{30}$ RNA editing is a molecular process effectively altering the amino acid sequence of the encoded protein to produce a new genomic sequence. It is crucial to develop "omics approach" for RNA editing, since RNA editing deals with modifying the nucleotide sequence in a specific genomic template to produce a new nucleotide sequence for understanding the molecular mechanisms of disease such as cancer. Deciphering these mutated sequences contributes to the development of disease-specific prognostic and therapeutic approaches. Different analytical methods have been employed for detailed analysis of genetic sequences such as gene chips, ${ }^{31}$ single-nucleotide polymorphism (SNP) microarrays, ${ }^{32}$ quantitative real-time polymerase chain reaction (qPCR), ${ }^{33}$ sanger gene sequencing, ${ }^{34}$ and so on. Among these, SNP microarray genotyping is a tool to determine genetic mutations, phenotypespecific panels, and genome-wide panels of a particular individual. ${ }^{32}$
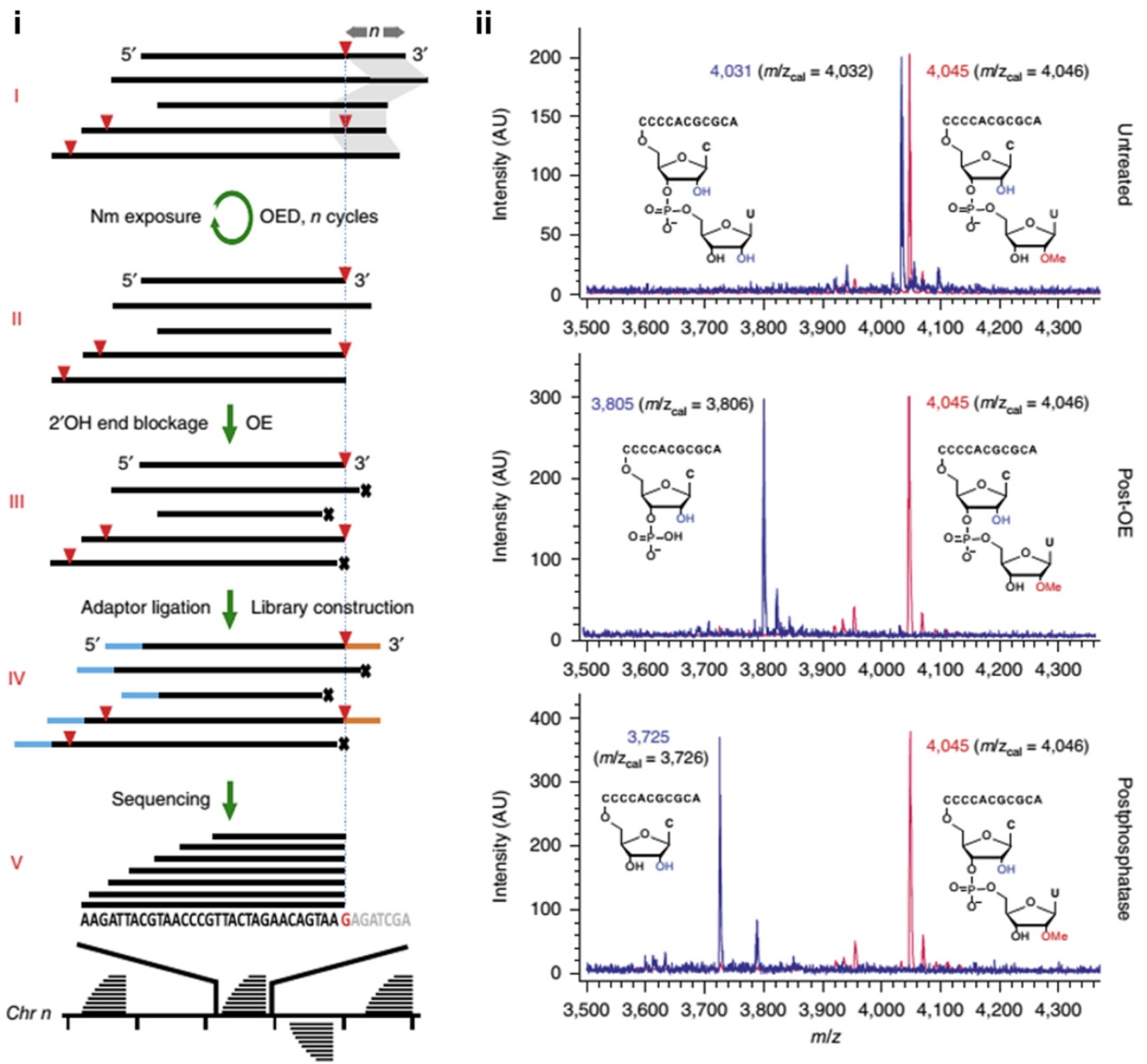

Figure 1. Mass spectrometry detection of nucleic acids for genetic analysis. Schematic illustration of Nm-seq method based on oxidative cleavage for mapping 2'-O-methylation with base precision. (i) was sequencing process and (ii) showed the obtained mass spectra. Reproduced with permission.[14] Copyright 2017, Springer Nature.

Combining MS with these microarray techniques provides the ability for rapid, accurate, and quantitative characterization of post-transcriptionally modified nucleosides 
based on their molecular weight changes. Hence, the continued improvements in the methodology and instrumentation used for the mass spectral analysis of nucleic acids will increase the applications of this technology to the field of genomics.

MS-based microarrays enable hybridization free analysis of nucleic acids based on their respective molecular weights. Research groups lead by Chuan $\mathrm{He}$ and Jianzhao Liu reported a new small molecule called as N6allyladenosine $\left(a^{6} \mathrm{~A}\right)$ for RNA (ribonucleic acid) labeling through both metabolic and enzyme-assisted manners (Figure 1). ${ }^{15}$

The total extracted RNAs were digested into single nucleosides and analyzed by ultra-highperformance liquid chromatography coupled
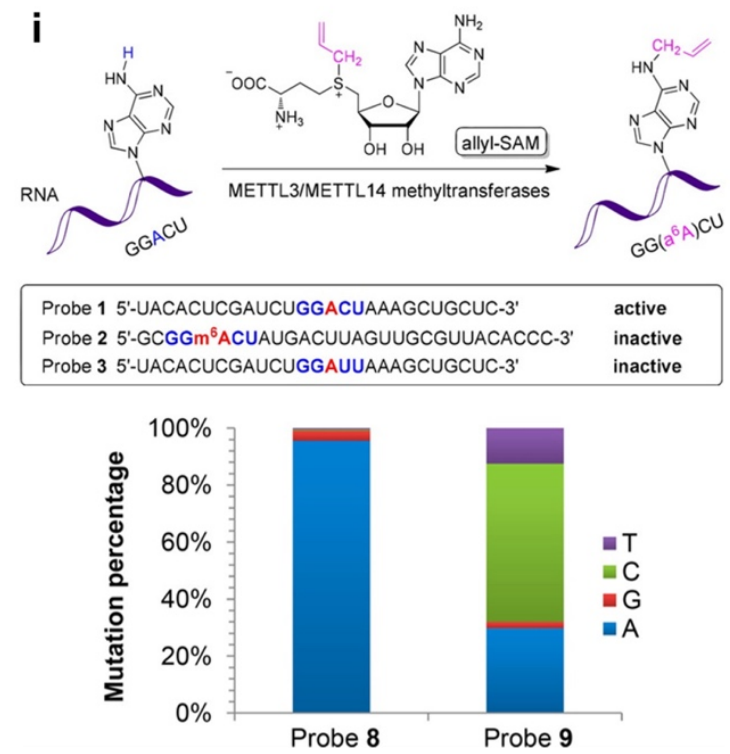

with triple quadrupole tandem mass spectrometer (UHPLC-QQQ-MS/MS) for accurate quantification of $\mathrm{a}^{6} \mathrm{~A}$ levels. Notably, the increased molar mass of a modified probe by MS quantification validated the proposed RNA post-treatment reaction mechanism. Overall, the metabolically incorporated $a^{6} \mathrm{~A}$ molecule provides facile differentiation of labeled and unlabeled RNA using reverse transcriptase (RT)-induced mutation assay for its potential sequencing applications in the RNA field. Research work by Dai et. al. utilized liquid chromatography-tandem mass spectrometry (LC-MS/MS) for accurate detection and quantification of 2'-O-methylated $(\mathrm{Nm})$ sites in mRNA molecules at low stoichiometry (Figure 2). ${ }^{14}$

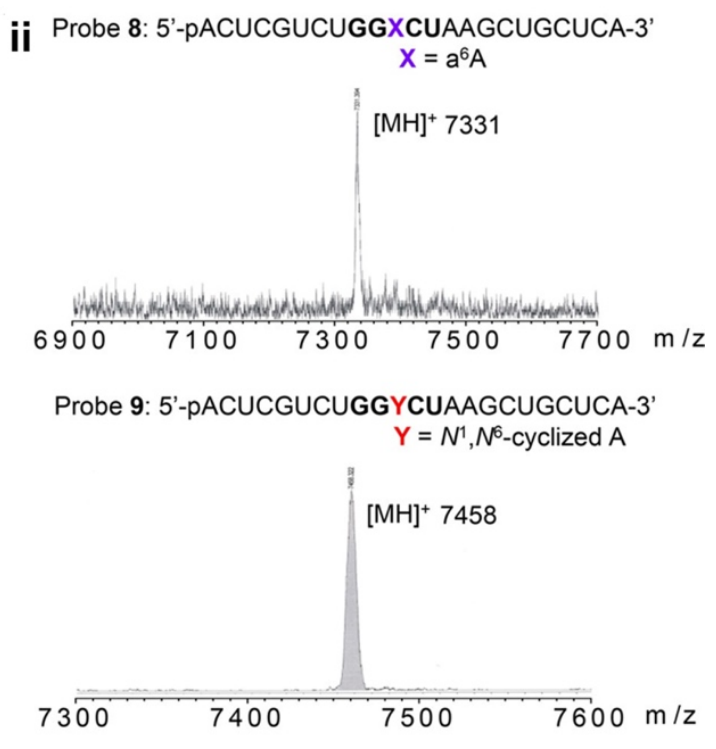

Figure 2. RNA labeling study for genetic analysis. (i) Test of the allyl transfer ability. (ii) Mass spectra of modified RNA oligo and percentages of mutation. Adapted with permission [15] Copyright 2017, American Chemical Society.

The MALDI-TOF spectra of modified and unmodified standard RNA oligonucleotides revealed the feasibility of RNA modification. A conceptually distinct approach was developed based on the different chemical properties of nucleosides with 2'-OH and 2'-OMe groups by periodate oxidation for exposing, enriching and mapping $\mathrm{Nm}$ sites in the transcriptome with single-nucleotide precision. Thus, deciphering the deregulated RNA processing events facilitates the detection of rare but functionally relevant transcripts at cellular level. The RNA editing studies will provide key insights for detecting disease-specific biomarkers and development of novel therapeutic strategies.

\section{Detection of proteins/peptides for proteomic analysis}

Proteomics focuses on the large-scale study of proteins produced or modified at the gene or cellular level by an organism. Proteomics is more complicated owing to the distinct gene expressions in every system. ${ }^{6,35,36}$ Proteins have been detected using variety of techniques such as antibody free/ labeled immunoassays, ${ }^{37}$ chromatographic techniques, ${ }^{38}$ or protein microarrays ${ }^{39}$ gel electrophoresis, ${ }^{40}$ and mass spectrometric methods prominently Orbitrap, matrix-assisted laser desorption/ionization (MALDI), and electrospray ionization 
(ESI). ${ }^{3,16,41}$ MS-based proteomics is an advanced technology interpreting encoded information in the genomes. $^{35,42,43}$ The approach has been successful in the case of small sets of proteins isolated in specific functional contexts. ${ }^{44}$ Detecting patterns of a differentially expressed proteins clinical samples shows the potential to diagnose the presence and stage of many diseases such as cancer. $^{35,45}$ Thus, the ability of MS to precisely identify and quantify thousands of proteins from complex samples has broadly impacted biology and medicine.

$\mathrm{Hu}$ and co-workers have developed a method using antibody-labeled and energy-focusing porous discoidal silicon nanoparticles (nanodisks) for detection of specific peptide fragments present in Mycobacterium tuberculosis (Mtb) using a high-throughput MS approach (Figure 3). ${ }^{16}$

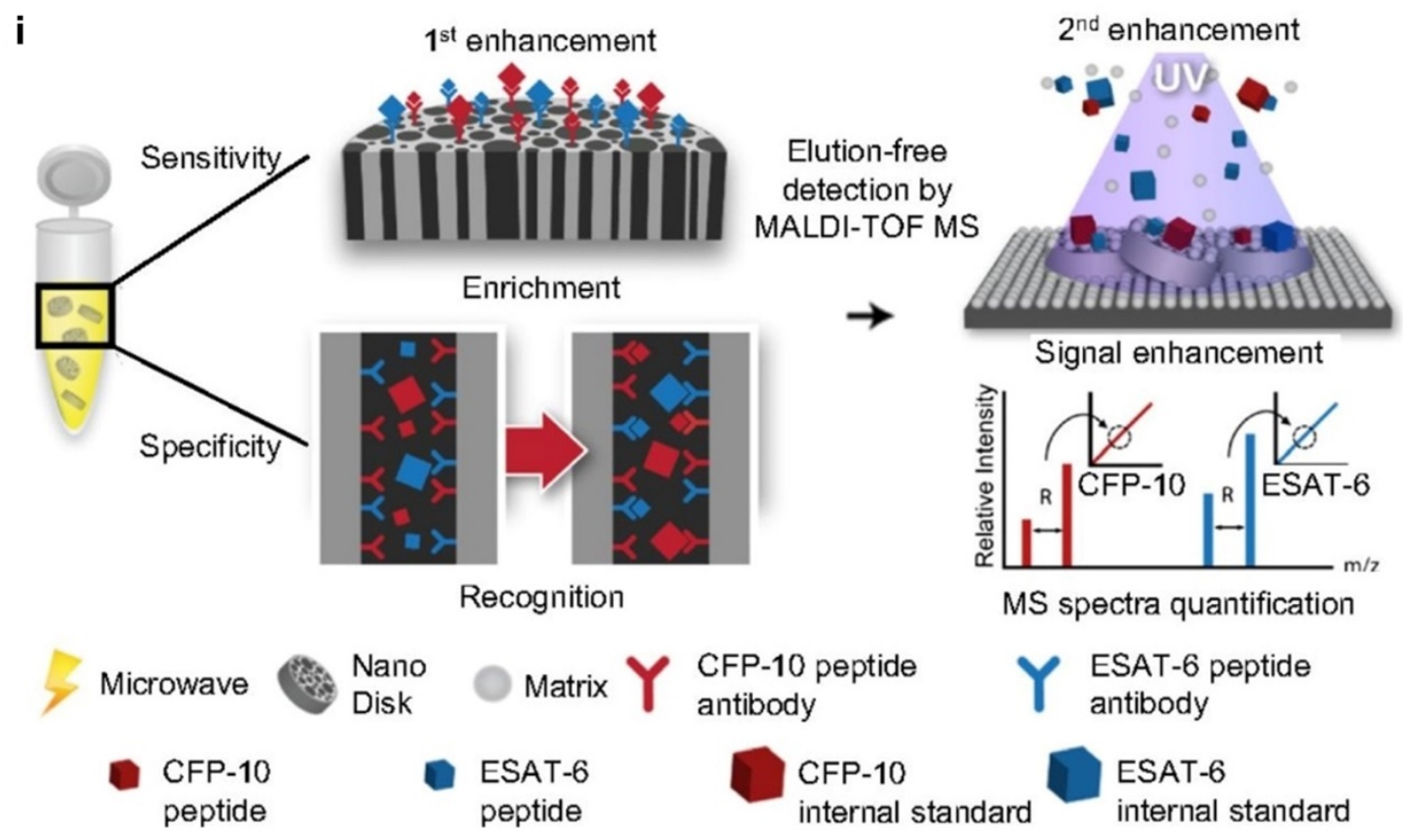

ii
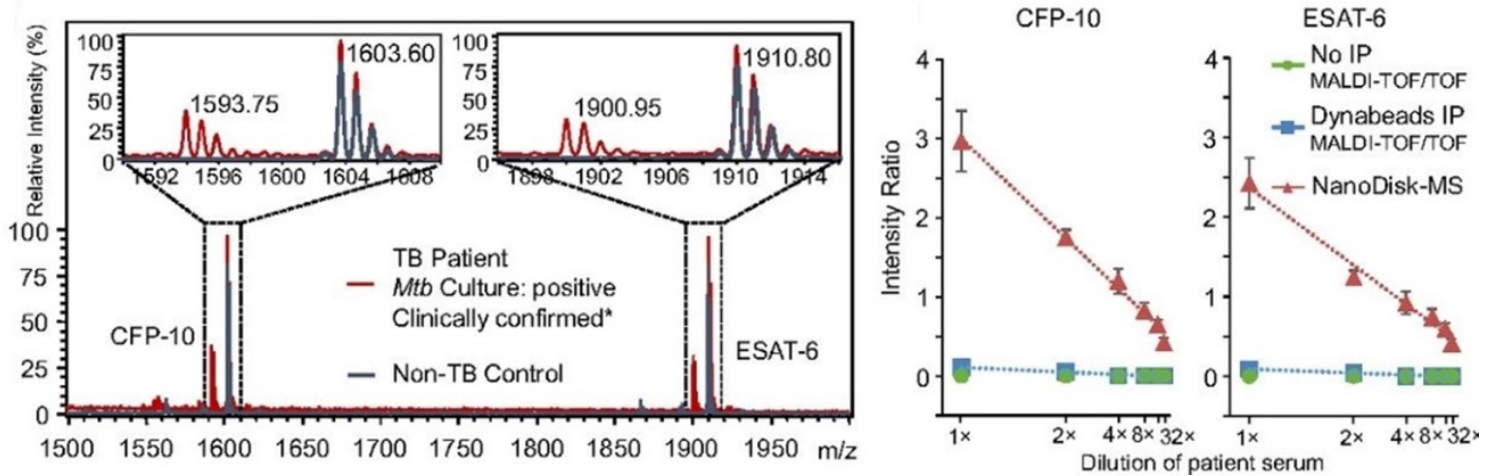

Figure 3. Mass spectrometry detection of proteins/peptides for proteomic analysis. The NanoDisk-MS platform. (i) Recognition and enrichment of target peptides and stable isotope-labeled internal standard peptides by antibody-conjugated nanodisks. (ii) Enhanced MS signals allowing quantification of target peptide at low concentrations. Reproduced with permission.[16] Copyright 2017, Proceedings of the National Academy of Sciences of the United States of America (PNAS) group.

NanoDisk-MS diagnosed active Mtb cases with robust sensitivity for cases of culture- positive pulmonary tuberculosis (PTB, 91.3\%) and extrapulmonary tuberculosis (EPTB, 
92.3\%). Another advanced technique known as mass cytometry by time-of-flight (CyTOF) has seen rapid developments of MS in proteomics. The CyTOF platform utilizes antibody coupled metal isotopes for mapping phenotypic heterogeneity and progression of cancer cells and hematologic malignancies. Lavin et. al. combined mass cytometry with single-cell transcriptomics and multiplex tissue imaging of the lung tumor for identifying natural killer and myeloid cell responses (Figure 4). ${ }^{17}$

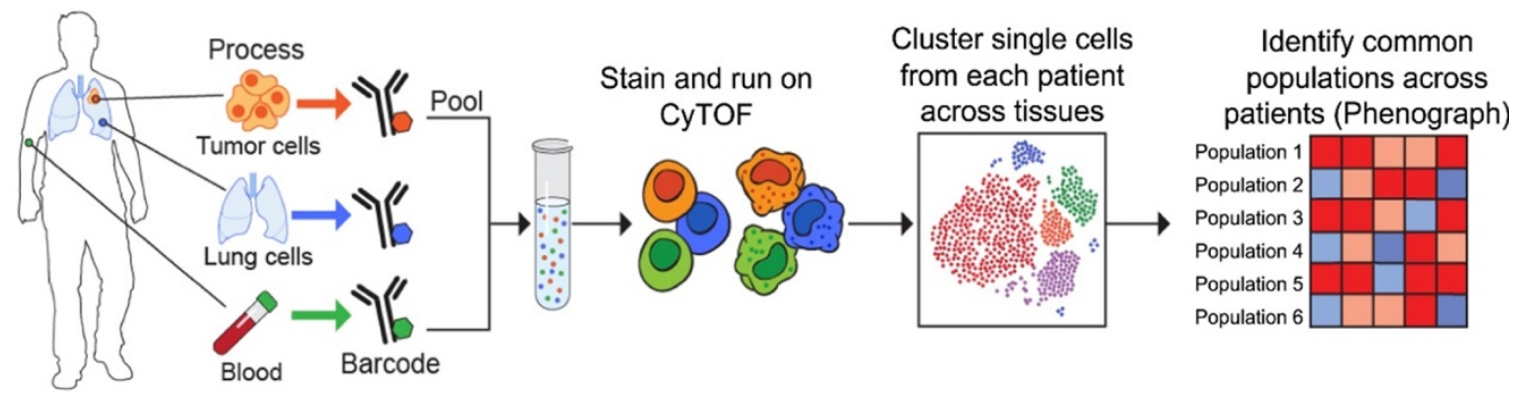

Surgical resection of primary lung tumors

Figure 4. Robust immune response to early lung adenocarcinoma tumor lesions and Schematic representation defining the immune composition of lung tumors. Mass cytometry (CyTOF) single-cell data was clustered to identify common populations across patients. Reproduced with permission.[17] Copyright 2017, Elsevier Inc.

Tumor lesions enriched in tertiary lymphoid structures had significantly more $\mathrm{T}$ lymphocytes and less macrophages as measured by CyTOF at the tumor site. Ginhoux and co-workers combined two high- dimensional technologies such as single-cell mRNA sequencing and CyTOF for identification of human blood $\mathrm{CD}_{123}{ }^{+} \mathrm{CD} 33^{+} \mathrm{CD} 45 \mathrm{RA}^{+}$dendritic cells precursors (pre-DC) (Figure 5). ${ }^{18}$
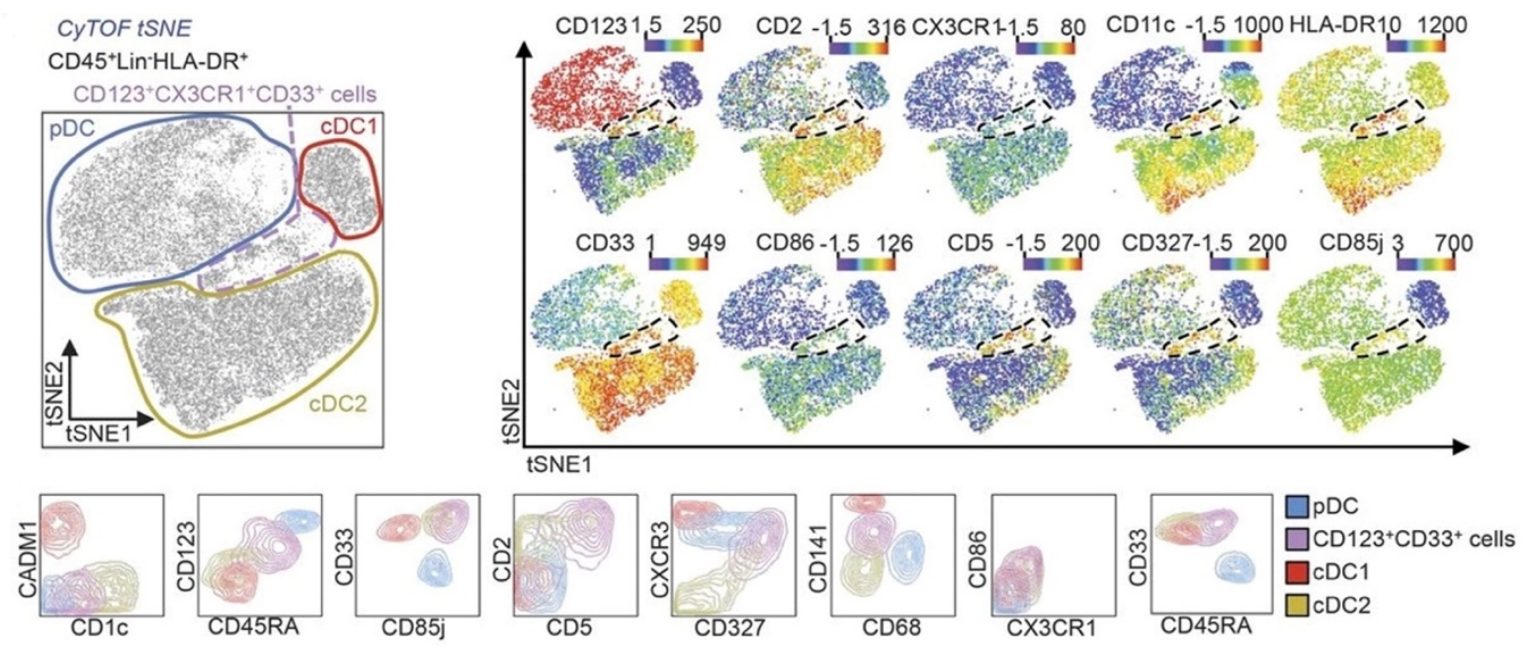

Figure 5. Mass cytometry (CyTOF) to identify rare CD123+CD33+ putative dendritic cell precursors (pre-DC). Reproduced with permission.[18] Copyright 2017, American Association for the Advancement of Science.

They unraveled the complexity of the human DC lineage at the single-cell level, revealing a continuous process of differentiation that starts in the bone marrow, and diverges at the point of emergence of pre-DC and plasmacytoid $\mathrm{DC}(\mathrm{pDC})$ potentials culminating in maturation of both lineages in the blood. Thus, the phenotypic heterogeneity in the circulating mature DC population can be evaluated by single-cell RNA sequence analysis, which was further confirmed by the marked dispersion in the t-stochastic neighbor embedding (tSNE) analysis of the CyTOF data. Even though MSbased proteomics is still an emerging technology, analysis of non-fragmented proteins with high accuracy has the potential to 
provide insights into detailed structural analysis.

\section{Detection of small molecules for metabolomics analysis}

The youngest emerging omics member, "metabolomics" has been considered important in early disease diagnosis and clinical evaluation. ${ }^{7,46}$ Analyzing the levels of all cellular metabolites from biological samples demonstrates the pathological and physiological status in biochemical pathway for biomarker identification. Identification of this distinctive fingerprinted metabolic pattern of an individual from the biological fluids could provide new insights for precision diagnostics. Compared to traditional biochemical analyzer, ${ }^{47}$ nuclear magnetic resonance spectrometry (NMR), ${ }^{48}$ and electrochemical methods $^{49}$ for metabolite analysis, MS techniques edge the unique advantages of desirable scanning speed, resolution, selectivity, sensitivity, and simple instrument structure over other methods in large-scale clinical applications. ${ }^{11,22}$ Notably, the use of matrices is of key significance in MS for laser energy transfer and desorption/ionization of analytes.

Qian research group developed a designer silver nanoshell ( $\left.\mathrm{SiO}_{2} @ \mathrm{Ag}\right)$ matrix for LDI MS assisted detection of small metabolites in cerebrospinal fluid (CSF) of patients with postoperative brain infection (Figure 6). ${ }^{20}$
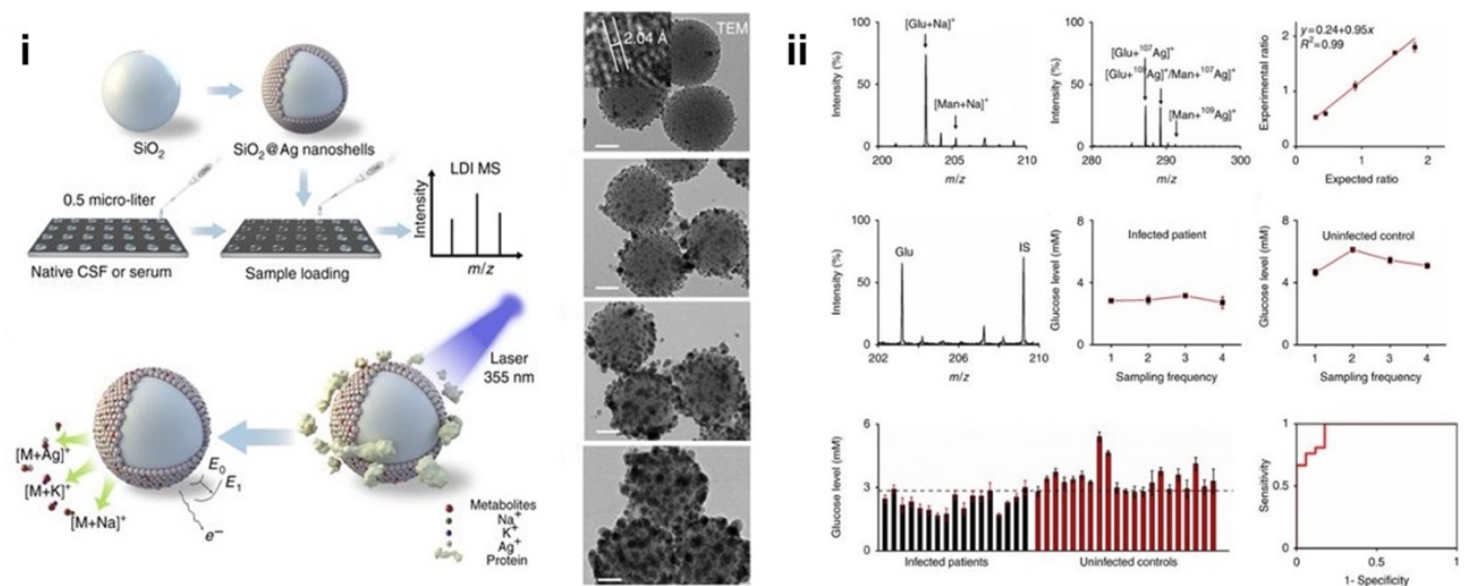

Figure 6. MS detection of small molecules for metabolic analysis. Plasmonic silver nanoshells for drug and metabolite detection. (i) Schematic diagrams of experimental workflow and LDI MS process using SiO2@Ag nanoshells as matrix. (ii) Diagnosis of postoperative brain infection based on glucose quantitation. Reproduced with permission. [20] Copyright 2017, Nature Publishing Group.

Isotopic quantification of selected metabolites revealed patients with postoperative brain infection by cerebrospinal fluid (CSF) analysis and monitored the drug concentrations in both CSF and serum to investigate the bloodbrain/CSF-barriers and for pharmacokinetics study. The same group also developed a novel plasmonic chip with gold nanoshells on the surface for in vitro metabolic diagnosis of early-stage lung cancer patients using serum and exosomes (Figure 7). ${ }^{19}$
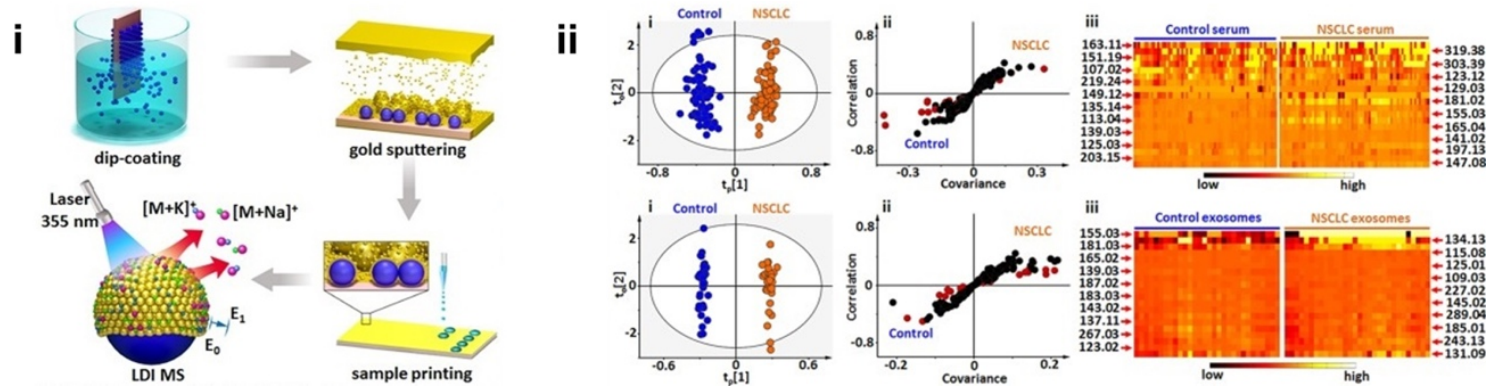

Figure 7. Metabolic fingerprinting on a gold chip. (i) Scheme of preparation and application of the plasmonic chip for LDI MS-based metabolic analysis. (ii) Diagnosis of early-stage cancer. Summary of LDI MS fingerprinting of serum and exosomes from early-stage cancer patients and healthy controls. Adapted with permission[19] Copyright 2018, American Chemical Society. 
The orthogonal partial least squares discriminant analysis (OPLS-DA) demonstrated clear group separation based on the fingerprinting results of serum and exosomes (both with $\mathrm{p}<0.0001$ ). Compared to the conventional MS methods for diagnosis of human cancer tissues, Eberlin and co-workers reported the development of an automated and biocompatible handheld mass spectrometry device named MasSpec Pen (Figure 8). ${ }^{21}$
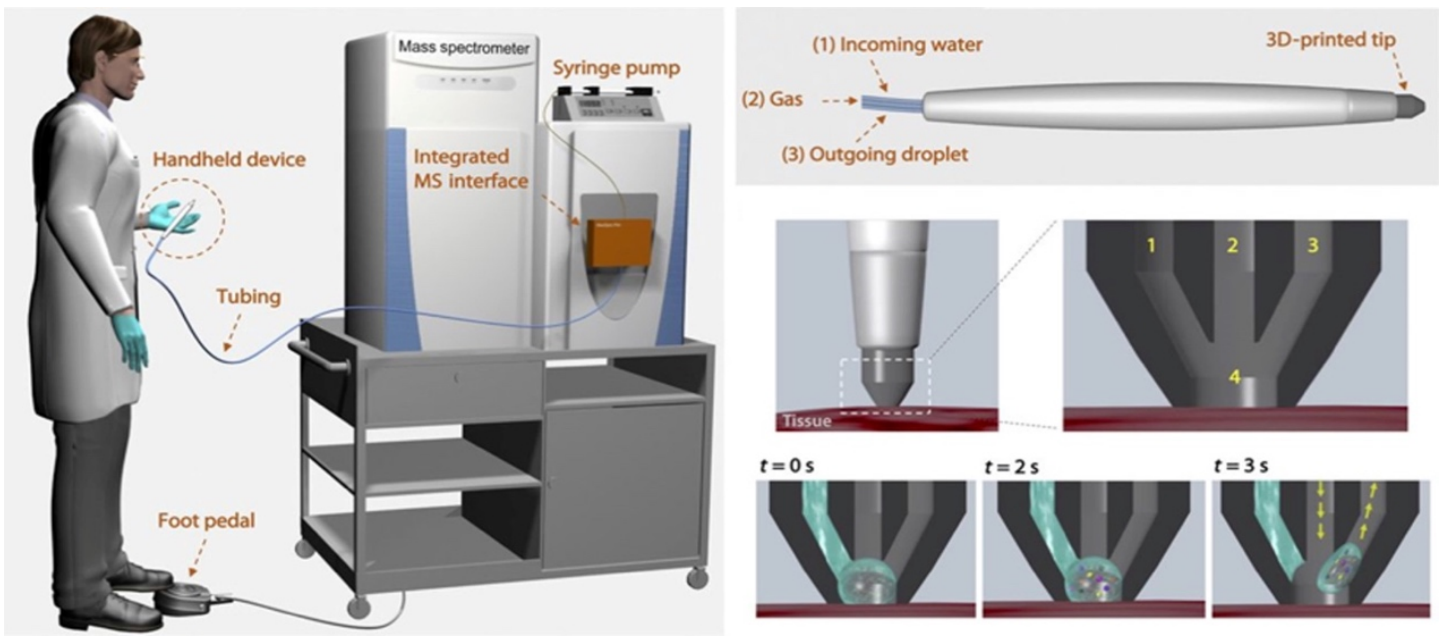

Figure 8. Schematic representation of the MasSpec Pen system and operational steps. The tip contacted the tissue for analysis, where the vacuum and the gas conduits were concomitantly opened (arrows) to transport the droplet from the MasSpec Pen to the mass spectrometer through the tubing system for molecular analysis. Reproduced with permission. ${ }^{[21]}$ Copyright 2017, American Association for the Advancement of Science.

The MasSpec Pen was utilized for molecular analysis of potential cancer biomarkers of normal and cancerous tissue samples from 253 patients. Statistical analysis technique allowed prediction of cancer with high sensitivity (96.4\%), specificity $(96.2 \%)$, and overall

\section{Conclusion}

In this review, we summarized the key milestones of MS, to address the needs from clinical omics towards precision diagnostics. In near future, research lines in the field would include but would not be limited to (1) integrated MS systems with low costs and smaller sizes; (2) high analytical performance for multi-omic analysis in one specimen; and (3) precision diagnosis of major diseases in large populations. Notably, considering the commercial value and social benefit of precision diagnostics, there would be emerging high-tech companies around the world to mine nano-assisted MS and facilitate the university application.

\section{Acknowledgments}

We gratefully thank the financial support from Project 81771983 and 81750110544 by National Natural Science Foundation of China (NSFC), Project 16441909300 by Shanghai Science and Technology Commission, Project 2017YFC0909000 by Ministry of Science and Technology of China. This work is also sponsored by the Program for Professor of Special Appointment (Eastern Scholar) at Shanghai Institutions of Higher Learning (TP2015015) and Innovation Research Plan supported by Shanghai Municipal Education Commission (ZXWF082101).

\section{Conflict of Interests}

The authors declare no conflicts of interest. For signed statements, please contact the journal office: editor@precisionnanomedicine.com 
Quote as: Li RX, Gurav DD, Wan JJ, Kun Qian K. A coming era of precision diagnostics based on nano-assisted mass spectrometry, Precis. Nanomed. 2018;1(2):162-172.

https://doi.org/10.33218/prnano1(2).180724.2

\section{References}

1 Mitchell, T. J. et al. Timing the Landmark Events in the Evolution of Clear Cell Renal Cell Cancer: TRACERx Renal. Cell 173, 611-+, doi:10.1016/j.cell.2018.02.020 (2018).

2 Ohman, E. M. et al. Clinically significant bleeding with low-dose rivaroxaban versus aspirin, in addition to P2Y12 inhibition, in acute coronary syndromes (GEMINI-ACS-1): a double-blind, multicentre, randomised trial. Lancet 389, 1799-1808, doi:10.1016/s0140-6736(17)30751-1 (2017).

3 Yusko, E. C. et al. Real-time shape approximation and fingerprinting of single proteins using a nanopore. Nature Nanotechnology 12, 360-367, doi:10.1038/nnano.2016.267 (2017).

4 Heath, J. R., Ribas, A. \& Mischel, P. S. Single-cell analysis tools for drug discovery and development. Nature Reviews Drug Discovery 15, 204-216, doi:10.1038/nrd.2015.16 (2016).

5 Woo, C.-W., Chang, L. J., Lindquist, M. A. \& Wager, T. D. Building better biomarkers: brain models in translational neuroimaging. Nature Neuroscience 20, 365-377, doi:10.1038/nn.4478 (2017).

6 Nakamura, A. et al. High performance plasma amyloid-beta biomarkers for Alzheimer's disease. Nature 554, 249-+, doi:10.1038/nature25456 (2018).

7 Mayerle, J. et al. Metabolic biomarker signature to differentiate pancreatic ductal adenocarcinoma from chronic pancreatitis. Gut 67, 128-137, doi:10.1136/gutjnl-2016-312432 (2018).

8 Manier, S. et al. Prognostic role of circulating exosomal miRNAs in multiple myeloma. Blood 129, 2429-2436, doi:10.1182/blood-2016-09-742296 (2017).

9 Kang, D.-K. et al. Rapid detection of single bacteria in unprocessed blood using Integrated Comprehensive Droplet Digital Detection. Nature Communications 5, doi:10.1038/ncomms6427 (2014).

10 Aebersold, R. \& Mann, M. Mass-spectrometric exploration of proteome structure and function. Nature 537, 347-355, doi:10.1038/nature19949 (2016).

11 Wu, J. et al. Multifunctional Magnetic Particles for Combined Circulating Tumor Cells Isolation and Cellular Metabolism Detection. Advanced Functional Materials 26, 4016-4025, doi:10.1002/adfm.201504184 (2016).

12 Schweppe, D. K. et al. Mitochondrial protein interactome elucidated by chemical cross-linking mass spectrometry. Proceedings of the National Academy of Sciences of the United States of America 114, 17321737, doi:10.1073/pnas.1617220114 (2017).

$13 \mathrm{Wu}, \mathrm{S}$. et al. A Plasmonic Mass Spectrometry Approach for Detection of Small Nutrients and Toxins. Nano-Micro Letters 10, doi:10.1007/s40820-018-0204-6 (2018).

14 Dai, Q. et al. Nm-seq maps 2 '-O-methylation sites in human mRNA with base precision (vol 14, pg 695, 2017). Nature Methods 15, 226-227, doi:10.1038/nmeth0318-226c (2018).

15 Shu, X. et al. N-6-Allyladenosine: A New Small Molecule for RNA Labeling Identified by Mutation Assay. Journal of the American Chemical Society 139, 17213-17216, doi:10.1021/jacs.7b06837 (2017).

16 Liu, C. et al. Quantification of circulating Mycobacterium tuberculosis antigen peptides allows rapid diagnosis of active disease and treatment monitoring. Proceedings of the National Academy of Sciences of the United States of America 114, 3969-3974, doi:10.1073/pnas.1621360114 (2017).

17 Lavin, Y. et al. Innate Immune Landscape in Early Lung Adenocarcinoma by Paired Single-Cell Analyses. Cell 169, 750-765, doi:10.1016/j.cell.2017.04.014 (2017).

18 See, P. et al. Mapping the human DC lineage through the integration of high-dimensional techniques. Science 356, doi:10.1126/science.aag3009 (2017).

19 Sun, X. et al. Metabolic Fingerprinting on a Plasmonic Gold Chip for Mass Spectrometry Based in Vitro Diagnostics. Acs Central Science 4, 223-229, doi:10.1021/acscentsci.7b00546 (2018).

20 Huang, L. et al. Plasmonic silver nanoshells for drug and metabolite detection. Nature Communications 8, doi:10.1038/s41467-017-00220-4 (2017).

21 Zhang, J. et al. Nondestructive tissue analysis for ex vivo and in vivo cancer diagnosis using a handheld mass spectrometry system. Science Translational Medicine 9, doi:10.1126/scitranslmed.aan3968 (2017). 
22 Stopka, S. A. et al. Molecular Imaging of Biological Samples on Nanophotonic Laser Desorption Ionization Platforms. Angewandte Chemie-International Edition 55, 4482-4486, doi:10.1002/anie.201511691 (2016).

23 Walkey, C. D., Olsen, J. B., Guo, H., Emili, A. \& Chan, W. C. W. Nanoparticle Size and Surface Chemistry Determine Serum Protein Adsorption and Macrophage Uptake. Journal of the American Chemical Society 134, 2139-2147, doi:10.1021/ja2084338 (2012).

24 Liu, B. et al. High Performance, Multiplexed Lung Cancer Biomarker Detection on a Plasmonic Gold Chip. Advanced Functional Materials 26, 7994-8002, doi:10.1002/adfm.201603547 (2016).

25 Sun, J., Xianyu, Y. \& Jiang, X. Point-of-care biochemical assays using gold nanoparticleimplemented microfluidics. Chemical Society Reviews 43, 6239-6253, doi:10.1039/c4cs00125g (2014).

26 Gan, J. et al. Designer SiO2@Au nanoshells towards sensitive and selective detection of small molecules in laser desorption ionization mass spectrometry. Nanomedicine-Nanotechnology Biology and Medicine 11, 1715-1723, doi:10.1016/j.nano.2015.06.010 (2015).

27 Sun, X., Wan, J. J. \& Qian, K. Designed Microdevices for In Vitro Diagnostics. Small Methods 1, doi:10.1002/smtd.201700196 (2017).

28 Zhang, J. X. et al. Predicting DNA hybridization kinetics from sequence. Nature Chemistry 10, 9198, doi:10.1038/nchem.2877 (2018).

29 Tian, X. et al. Circulating tumor DNA 5-hydroxymethylcytosine as a novel diagnostic biomarker for esophageal cancer. Cell Research 28, 597-600, doi:10.1038/s41422-018-0014-x (2018).

30 Peng, X. et al. A-to-I RNA Editing Contributes to Proteomic Diversity in Cancer. Cancer Cell 33, 817-+, doi:10.1016/j.cell.2018.03.026 (2018).

31 Jung, C. et al. Massively Parallel Biophysical Analysis of CRISPR-Cas Complexes on Next Generation Sequencing Chips. Cell 170, 35-+, doi:10.1016/j.cell.2017.05.004(2017).

32 Ehret, G. B. et al. The genetics of blood pressure regulation and its target organs from association studies in 342,415 individuals. Nature Genetics 48, 1171-1184, doi:10.1038/ng.3667 (2016).

33 Liu, Q. et al. Circulating exosomal microRNAs as prognostic biomarkers for non-small-cell lung cancer. Oncotarget 8, 13048-13058, doi:10.18632/oncotarget.14369 (2017).

34 Hayashi, G. et al. Base-Resolution Analysis of 5-Hydroxymethylcytosine by One-Pot Bisulfite-Free Chemical Conversion with Peroxotungstate. Journal of the American Chemical Society 138, 14178-14181, doi:10.1021/jacs.6b06428 (2016).

35 Khodadoust, M. S. et al. Antigen presentation profiling reveals recognition of lymphoma immunoglobulin neoantigens. Nature 543, 723-+, doi:10.1038/nature21433 (2017).

36 Chen, I. H. et al. Phosphoproteins in extracellular vesicles as candidate markers for breast cancer. Proceedings of the National Academy of Sciences of the United States of America 114, 3175-3180, doi:10.1073/pnas.1618088114 (2017).

37 Hansen, C. H., Yang, D., Koussa, M. A. \& Wong, W. P. Nanoswitch-linked immunosorbent assay (NLISA) for fast, sensitive, and specific protein detection. Proceedings of the National Academy of Sciences of the United States of America 114, 10367-10372, doi:10.1073/pnas.1708148114 (2017).

38 Ismail, H. M. et al. Artemisinin activity-based probes identify multiple molecular targets within the asexual stage of the malaria parasites Plasmodium falciparum 3D7. Proceedings of the National Academy of Sciences of the United States of America 113, 2080-2085, doi:10.1073/pnas.1600459113 (2016).

39 Sims, R. et al. Rare coding variants in PLCG2, ABI3, and TREM2 implicate microglial-mediated innate immunity in Alzheimer's disease. Nature Genetics 49, 1373-+, doi:10.1038/ng.3916 (2017).

40 de Bruin, G., Xin, B.-T., Florea, B. I. \& Overkleeft, H. S. Proteasome Subunit Selective ActivityBased Probes Report on Proteasome Core Particle Composition in a Native Polyacrylamide Gel Electrophoresis Fluorescence-Resonance Energy Transfer Assay. Journal of the American Chemical Society 138, 9874-9880, doi:10.1021/jacs.6b04207 (2016).

41 Rejeeth, C. et al. Extraction, detection, and profiling of serum biomarkers using designed Fe3O4@SiO2@HA core-shell particles. Nano Research 11, 68-79, doi:10.1007/s12274-017-1591-6 (2018).

42 Xiao, H., Suttapitugsakul, S., Sun, F. \& Wu, R. Mass Spectrometry-Based Chemical and Enzymatic Methods for Global Analysis of Protein Glycosylation. Accounts of chemical research, doi:10.1021/acs.accounts.8b00200 (2018). 
$43 \mathrm{Li}$, Y. et al. High-efficiency nano/micro-reactors for protein analysis. Rsc Advances 5, 1331-1342, doi:10.1039/c4ra12333f (2015).

44 Qian, K., Zhou, L., Zhang, J., Lei, C. \& Yu, C. A combo-pore approach for the programmable extraction of peptides/proteins. Nanoscale 6, 5121-5125, doi:10.1039/c4nr00633j (2014).

45 Borrebaeck, C. A. K. Precision diagnostics: moving towards protein biomarker signatures of clinical utility in cancer. Nature Reviews Cancer 17, 199-204, doi:10.1038/nrc.2016.153 (2017).

46 Patti, G. J., Yanes, O. \& Siuzdak, G. Metabolomics: the apogee of the omics trilogy. Nature Reviews Molecular Cell Biology 13, 263-269, doi:10.1038/nrm3314 (2012).

47 Ziv, C. et al. Viral serine palmitoyltransferase induces metabolic switch in sphingolipid biosynthesis and is required for infection of a marine alga. Proceedings of the National Academy of Sciences of the United States of America 113, E1907-E1916, doi:10.1073/pnas.1523168113 (2016).

48 Hattori, A. et al. Cancer progression by reprogrammed BCAA metabolism in myeloid leukaemia. Nature 545, 500-+, doi:10.1038/nature22314 (2017).

49 Pappa, A. M. et al. Direct metabolite detection with an n-type accumulation mode organic electrochemical transistor. Science advances 4, eaat0911-eaat0911, doi:10.1126/sciadv.aat0911 (2018). 